\title{
Magnetic Resonance Energy Transmission and Driving System of Micro Robot
}

\author{
Aobo Cui \\ School of Electronic Information Engineering, Beijing Jiaotong University, Beijing, China
}

Keywords: Micro robots, Magnetic resonance, Wireless power transmission, Efficiency characteristic

\begin{abstract}
Magnetic resonance type wireless power transmission technology is a new development direction of wireless power transmission technology. Energy is transferred between coils through resonance and coupling of non-radiation near-region magnetic field. This paper analyzes the influencing factors of the transmission power of the magnetic resonance wireless energy transmission system of the micro robot. Based on the comparison of the transmission power characteristics of the non-resonant and resonant systems, the analysis idea of overall analysis of the energy transmission system loss is put forward, and the capacitance loss and the drive source loss are included in the analysis scope. According to the characteristic that the peak power and peak efficiency operating frequency points do not coincide, an energy efficiency optimization control strategy based on information feedback is proposed. With the aid of energy transmission channel, the working state of the receiving end is fed back to the transmitting end in real time by using load modulation technology to realize closed-loop control of the energy transmission process. The experiment verifies the feasibility of the power amplifier in the magnetic resonance wireless power transmission system and significantly improves the transmission efficiency of the wireless power transmission system.
\end{abstract}

\section{Introduction}

Wireless power transmission technology is a technology in which electrical equipment obtains energy from the power supply in a non-contact manner. It realizes complete electrical isolation between the power supply and the electrical equipment. The transmission process is safe, reliable and flexible, which provides the possibility for human beings to get rid of the shackles of wires [1]. When a rechargeable battery is used, the used battery needs to be detached from the micro-robot and reassembled after charging before it can be put into use again, which causes the interruption of the work of the micro-robot. The basic structure of the technology uses coupling coils as the main components of energy transmission, and uses the resonance effect between resonance coils to improve the output power. Due to the long distance between coils, resonance, loose coupling and other factors. The transmission distance of magnetic resonance type is longer than that of magnetic induction type. It realizes high-efficiency energy transmission through magnetic coupling resonance of transmitting coil and receiving coil [2], but the transmission power is relatively low. It is mainly used in fields such as wireless charging equipment and consumer electronic equipment in smart homes [3]. Wireless power transmission technology integrates many key technologies such as power electronics, electromagnetic field, physics, control and so on, and has important theoretical and application values. As a new interdisciplinary research field, this technology has received extensive attention from academia and industry at home and abroad.

\section{System Model}

Fig. 1 is an equivalent circuit model of a resonant system based on mutual inductance coupling theory. In the figure: $L_{1}, L_{2}, L_{3}$ and $L_{4}$ are the self-inductance of the drive, transmit, receive and pick-up coils respectively. $C_{2}$ and $C_{3}$ are resonance capacitances of the transmitting coil and the receiving coil, respectively. The principle of contactless energy transmission is very similar to the 
working principle of transformer. The main difference lies in low magnetization inductance and leakage inductance. Energy is converted from single-phase power frequency AC power supply to DC power after rectification, and then converted to high-frequency voltage by high-frequency drive source. For example, the swing of a simple pendulum, the movement of a spring oscillator, etc [4]. To solve this kind of mechanical vibration, the motion equation of the system must be listed according to the mechanical theory, and the circuit equation of the resonant circuit has the same form as some vibration equations. $R_{1}, R_{2}$ and $R_{3}$ are equivalent resistances of driving, transmitting and receiving coil loops respectively; $R_{L}$ is the equivalent load of the system, including the resistance of the pickup coil; $\dot{V}_{1}$ is the excitation voltage source; $M_{12}, M_{23}$ and $M_{34}$ are mutual inductance between the two loops.

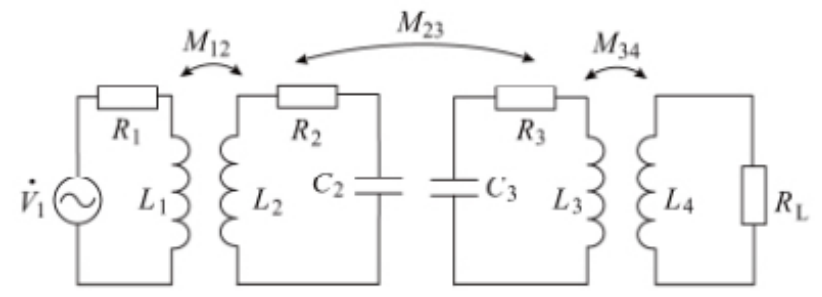

Fig.1 Equivalent Circuit Model of Resonant System

\section{Analysis of Power and Efficiency Transmission Characteristics}

\subsection{Power Transmission Characteristics}

Fig. 2 is a typical frequency response curve of load power transmission characteristics of a magnetic resonance coupled wireless power transmission system. It can be seen that for the general magnetic resonance coupling wireless energy transmission system, there is a difference in the master-slave resonance frequency under the condition of bifurcation of resonance frequency. Contactless power transmission technology is very suitable for supplying power to moving loads (e.g. electric vehicles). Through magnetic field coupling, it is transmitted to the secondary coupling coil and then flows through the secondary matching tuning circuit to be supplied to the load circuit. It can be seen that the energy contained in both circuits oscillates with time (which can be determined by the magnitude of the current peak). The load power at the main resonance frequency increases first and then decreases. Because the planar spiral coil has higher coupling coefficient and quality factor than other structural coils, it is more suitable for wireless power transmission [5]; Therefore, the maximum load power of the system is not obtained under the condition of large coupling coefficient at the transceiver end, and the maximum load power transmission may occur under the condition of large distance between the transceiver end.

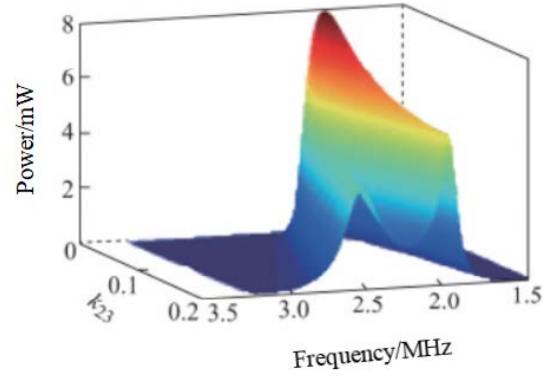

Fig.2 The Relationship between Load Power and Working Frequency and $k_{23}$ 


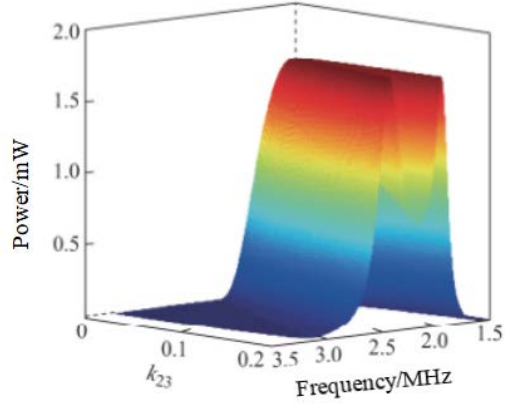

Fig.3 The Relationship between Load Power and Operating Frequency and $k_{23}$ When the Parameters of Transceiver Are the Same

The transmission system with exactly the same parameters at the transmitting end (driving and transmitting coil loops) and the receiving end (receiving and picking coil loops) is a special case. The system has its own particularity besides the general characteristics of magnetic resonance coupling transmission system. The flux density is proportional to the excitation current. Increasing excitation current can increase induced voltage, but this method increases wire diameter and does not make full use of iron core. The output power is proportional to the square of the coupling coefficient and the effective value of the input voltage. Increasing the coupling coefficient and the input voltage is beneficial to increasing the output power. The coupling coefficient can quantitatively describe the coupling tightness of the two coils, that is, the transmission distance of the system, which is defined as the ratio of the mutual inductance flux linkage of the two coils to the coil self-inductance flux linkage. In order to calculate the fixed size of the resonant coil when the power transmission efficiency is maximum, researchers have designed the self-resonant coil with flexible coaxial capacitance and the single circular resonant ring with flat capacitance [6]. Fig. 3 is a simulation diagram of the load power frequency response curve under the condition of the same transceiver parameters.

\subsection{Efficiency Transmission Characteristic}

Fig. 4 is a graph showing the variation of system transmission efficiency with operating frequency and k23 under the parameters of fig. 3. It can be seen that the variation of transmission efficiency with operating frequency and coupling coefficient K23 is similar to the load power transmission curve. At this time, the output power is only related to the coupling coefficient, input voltage and output impedance. Coupling coefficient is restricted by coil shape, size, distance and other factors. Frequency splitting meets the maximum power transmission condition. The boundary between the under-coupled region and the over-coupled region is the critical coupling region. The maximum point of load power in this region is called the critical coupling extreme point, which corresponds to the maximum transmission distance under the condition of maximum power transmission.

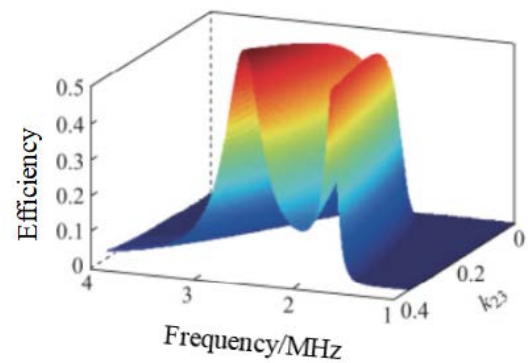

Fig.4 The Relationship between Transmission Efficiency and Working Frequency and $k_{23}$

The four-coil system can be equivalent to a double-coil resonance system considering the influence of drive and pick-up coils. The solution of the critical coupling coefficient of the doublecoil in Reference [7] shows that the critical coupling coefficient of the four-coil resonance system is: 


$$
k_{c}=\left[\frac{\left(R_{1}^{\prime}+R_{2}\right)^{2}+\left(R_{L}^{\prime}+R_{3}\right)^{2}}{2 \omega_{r}^{2} L_{2}^{2}}\right]^{\frac{1}{2}}
$$

Where $\omega_{r}$ is the natural resonant frequency of the transmitting coil and the receiving coil.

For a mr system determined by each coil parameter, there is an optimal relative position at the transmitting and receiving ends, i.e. there is an optimal value for the coupling coefficient $k_{23}$ between the transmitting coil and the receiving coil. When the system operates under this coupling coefficient, the load will obtain the maximum power. Then the load power can be approximately expressed as:

$$
P_{0} \approx \frac{\omega_{r}^{2} M_{23}^{2} V_{2}^{2} R_{L}^{\prime}}{2\left[\left(R_{2}+R_{1}^{\prime}\right)\left(R_{3}+R_{L}^{\prime}\right)+\omega_{r}^{2} M_{23}^{2}\right]^{2}}
$$

Where: $V_{2}$ is the voltage amplitude of the equivalent excitation voltage source of the transmission coil.

When $d P_{0} / d k_{23}=0$, the optimal coupling coefficient $k_{23-o p t}$ of the system under the maximum load power transmission target is obtained as follows:

$$
k_{23-o p t} \approx \sqrt{\frac{\left(R_{2}+R_{1}^{\prime}\right)\left(R_{3}+R_{L}^{\prime}\right) C_{2}}{L_{3}}}
$$

Based on this idea, the finite element analysis of the magnetic field distribution under the structure of 2-7 wires is carried out. A frequency tracking control method in which the working frequency of the transmitting power source synchronously tracks the natural resonant frequency of the transmitting circuit, thereby avoiding $\mathrm{mr}$ system detuning and greatly improving the transmission efficiency [8]. And with the increase of the distance between the transmitting and receiving ends, the two frequencies converge together, and the phenomenon of frequency bifurcation disappears. There is no obvious difference between the efficiency characteristic curves under the condition of whether the parameters of the transceiver are symmetrical or not.

\section{Peak Power and Peak Efficiency Transmission}

For a wireless power transmission system, it is hoped to obtain the desired transmission effect through reasonable parameter design. Load power and transmission efficiency are two important indexes of system transmission, and peak power transmission or peak efficiency transmission are the effects that designers often hope to achieve. The interaction between various parameters and the design of each parameter need to be studied by finite element analysis. By introducing resonance compensation, a resonant coil with a higher quality factor can still obtain a larger input power for the second time and realize a higher efficiency of wireless energy transmission [9]. The critical coupling extreme point is the ideal working point of the system. At this point, the system can realize the farthest transmission distance under the premise of ensuring the maximum power transmission. The purpose of matching is to make the system work at the critical coupling extreme point. Fig. 5 is a variation curve of the system peak power and the corresponding operating frequency when the peak efficiency is transmitted at different relative positions of the transceiver, and the simulation parameters are the same as those in fig. 4. 


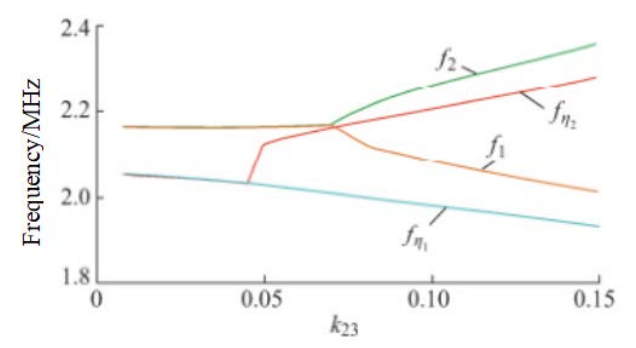

Fig.5 The Frequency At the Peak Power and Efficiency of the System Varies with $k_{23}$

Table 1 Peak Power And Peak Efficiency Transmission

\begin{tabular}{|l|l|l|l|l|l|l|}
\hline Situation & $f_{0} / \mathrm{MHz}$ & $f_{\eta} / \mathrm{MHz}$ & $P_{1} / \mathrm{mW}$ & $P_{2} / \mathrm{mW}$ & $\eta_{1} / \%$ & $\eta_{2} /$ \\
\hline 1 & 1.88 & 2.3 & 50.19 & 47.71 & 40.66 & 42.91 \\
\hline 2 & 1.58 & 2.17 & 40.29 & 27.08 & 39.71 & 40.08 \\
\hline 3 & 1.93 & 1.86 & 33.24 & 30.91 & 51.00 & 51.71 \\
\hline 4 & 1.94 & 2.06 & 35.17 & 24.03 & 46.32 & 47.34 \\
\hline
\end{tabular}

Table 1 lists the peak power $P_{1}$ of the system and the transmission efficiency $\eta_{1}$ under this condition, and also lists the experimental values of the peak transmission efficiency $\eta_{2}$ and the load power $P_{2}$ under this condition. In the table: $f_{0}$ and $f_{\eta}$ are the system operating frequencies when peak power and peak efficiency are transmitted respectively; Iron core is a very important element for charger, not only because it supports the winding of pickup coil, but also because it provides a low reluctance path. Reducing the resistance is beneficial to reducing the active loss of the system. The characteristic that the peak power and peak efficiency of the transmission system do not coincide with each other is similar to the characteristic that the maximum load power and efficiency of the linear active two-terminal network are obtained under different load conditions.

\section{Energy Efficiency Optimization Control}

\subsection{Information Reverse Transmission Technology}

The transmission performance of magnetic resonance wireless energy transmission system is affected by all parameters. The change of load resistance will cause the change of loop equivalent impedance, thus affecting the loop current amplitude of the coil, i.e. modulation. Coil resistance is mainly affected by two factors, one is the diameter and material of coil wire; The other is that the influence of skin effect and proximity effect cannot be ignored because the conductor works at high frequency. As long as splitting occurs, the splitting peaks all meet the maximum power transmission conditions, and their heights are the same, and are equal to the heights of critical coupling extreme points, but the critical coupling extreme points are all the points that meet the maximum power transmission conditions, and the corresponding coupling coefficients are the smallest. The load is short-circuited and cannot receive power, but this period accounts for a small proportion of the total power transmission time. One of the most critical problems is that the system power transmission efficiency is very low. Although new technologies are adopted in practical electronic equipment, energy is greatly wasted [10]. The usual filter capacitor flows only ripple current, and the current is small. However, since the resonant capacitor works in a resonant state, the capacitor will repeatedly flow through the charging current and continuously exchange reactive energy with the power supply and coil. That is, more load power can be obtained, but the effective transmission distance will be reduced. Reducing the matching factor has the opposite effect.

\subsection{Experimental Verification}

Adaptive adjustment between transmission of value power or peak efficiency. In some applications, according to the working stage of the system, the energy transmitting end control circuit adjusts the frequency of the excitation signal in the input drive coil to switch between peak 
power transmission and peak efficiency transmission. The capacitor must be a high voltage capacitor. At the same time, although improving the quality factor of the resonant circuit is beneficial to improving the transmission power, a larger quality factor means that only a high voltage capacitor with a smaller capacity can be selected after parameter design. What needs to be explained here is that this design principle is designed for radio frequency circuits. Its purpose is to obtain greater transmission power and reduce the power reflected back to the source. However, the power consumed by the internal resistance of the source itself is generally not considered. According to the maximum power transmission theorem, when the load matches, the load receives the largest power. When a transistor is used as a switch, it usually has two working states: one is saturation conduction, which is equivalent to "switch on" because its saturation conduction voltage drop is very small (or internal resistance is very small); The energy transmitting end controller adjusts the signal frequency generated by the signal generator to change the excitation signal frequency in the transmission line loop; With the help of information feedback technology, the energy receiving end periodically samples the received power of the load in real time through the power detection module. When selecting capacitor elements, it is necessary to meet the conditions of high frequency, large current, high withstand voltage, small capacitance, small volume and low equivalent series resistance at the same time, so the characteristics of capacitors are required to be high.

\section{Conclusion}

In this paper, the magnetic resonance wireless power transmission system is studied. Based on the vibration theory and mutual inductance coupling theory, the system is analyzed, and the finite element software HFSS is used to simulate the system. Finally, the experimental verification is carried out. Under loose coupling condition, improving the quality factor of resonance can increase the transmission efficiency. The shape of the load power frequency response curve is closely related to the value of the transmission parameters. The load power frequency response curve is symmetrical for systems with the same parameters at the transceiver end. Based on the maximum power transmission theorem, the frequency characteristics of the system are analyzed in detail, and the splitting frequency and splitting peak value are obtained. The working states of the system in the over-coupling region, the critical coupling region and the under-coupling region are studied, and it is concluded that when the system works at the critical coupling extreme point. Both the load power and the transmission efficiency of the resonant system may increase first and then decrease with the increase of the distance between the transmitting and receiving ends. When selecting capacitor elements, it is necessary to meet the conditions of high frequency, large current, high withstand voltage, small capacitance, small volume and low equivalent series resistance at the same time, so the characteristics of capacitors are required to be high.

\section{References}

[1] Xu Dong, Wang Dongqing, Cheng Jie. (2017). Analysis of resonance frequency of magnetic resonance wireless energy transmission system. Journal of Qingdao University: Engineering and Technology Edition, no. 4, pp. 1-5.

[2] Gong Xiuyuan, Wang Dongqing. (2019). Design of Integral Sliding Mode Control in MR Wireless Power Transmission System. Journal of Qingdao University (Engineering Technology Edition), vol. 034, no. 003, pp. 1-6.

[3] Li Zhiyong, Zhou Fangyi, Li Xiaoguang, et al. (2017). Efficiency analysis of wireless energy transmission system for PCB magnetic resonance coils. High Power Converter Technology, no. 3, pp. 63-68.

[4] Wang Huizhong, Zhuang Yajie, Xue Chao. (2019). Research on LCC-P resonant wireless energy transmission system. Electronic Design Engineering, vol. 027, no. 014, pp. 20-25. 
[5] Fan Xingming, Jia Erju, Zhang Xin. (2018 ). Load characteristic analysis and coil parameter design of MCR-WPT system. High Voltage Apparatus, no. 1, pp. 29-34.

[6] Li Xiaopeng, Yuan Xueqing, Xu Kailin. (2017). Research and implementation of a medium power wireless energy transmission system equipment. Science Discovery, vol. 5, no. 2, pp. 151156.

[7] Mark, Mao Jialuo, Wang Yunjie, et al. (2017). Research on magnetic resonance wireless power transmission technology. Intelligent Robot, no. 2, pp. 61-63.

[8] Liu Suqi, Tan Jianping, Literature. (2017). Dynamic impedance compensation mechanism and implementation method of wireless energy transmission system. Industrial and Mining Automation, vol. 43, no. 12, pp. 36-43.

[9] Shi Xiaokun, Li Yongkun, Guo Qinggong. (2019). Miniaturization design of a three-coil magnetic resonance wireless energy transmission system. Journal of Sichuan University: Natural Science Edition, no. 4, pp. 671-676.

[10] Liu Suqi, Tan Jianping, Wen xue, et al. (2017). Multi-inverter power synthesis mechanism of wireless energy transmission system. Industrial and Mining Automation, vol. 43, no. 1, pp. 60-65. 